\title{
Theory of the Phonon Hall Effect in Paramagnetic Dielectrics
}

\author{
L. Sheng, ${ }^{1}$ D. N. Sheng, ${ }^{2}$ and C. S. Ting ${ }^{1}$ \\ ${ }^{1}$ Department of Physics and Texas Center for Superconductivity, University of Houston, Houston, Texas 77204, USA \\ ${ }^{2}$ Department of Physics and Astronomy, California State University, Northridge, California 91330, USA
}

(Received 13 January 2006; published 18 April 2006)

\begin{abstract}
Based upon Raman spin-lattice interaction, we propose a theoretical model for the phonon Hall effect in paramagnetic dielectrics, which was discovered recently in an experiment [C. Strohm, G. L. J. A. Rikken, and P. Wyder, Phys. Rev. Lett. 95, 155901 (2005).]. The phonon Hall effect is revealed to be a phonon analogue to the anomalous Hall effect in electron systems. The thermal Hall conductivity is calculated by using the Kubo formula. Our theory reproduces the essential experimental features of the phonon Hall effect, including the sign, magnitude, and linear magnetic field dependence of the thermal Hall conductivity.
\end{abstract}

PACS numbers: 66.70.+f, 72.10.Bg, 72.15.Gd, 72.20.Pa

When an electrical current flows through a conductor with direction perpendicular to an applied magnetic field, a transverse electrical current may be generated in the third perpendicular direction. This is well known as the Hall effect, and is due to the electromagnetic Lorentz force on the charge carriers. Two interesting variants of the conventional Hall effect are the anomalous Hall effect (AHE) in ferromagnets [1] and the spin Hall effect in nonmagnetic conductors [2], where the electron spin-orbit coupling plays an essential role. The AHE is characterized by an anomalous contribution to the Hall resistivity proportional only to the magnetization. Intuitively, one would not expect a Hall effect for phonons, which do not carry charges and do not couple to the magnetic field directly. Remarkably, by applying a magnetic field perpendicular to a heat current flowing through a sample of the paramagnetic dielectric $\mathrm{Tb}_{3} \mathrm{Ga}_{5} \mathrm{O}_{12}$, Strohm, Rikken, and Wyder observed very recently a temperature difference of up to $200 \mu \mathrm{K}$ between the sample edges in the third perpendicular direction [3]. The temperature difference is attributed to the phonon Hall effect (PHE) [3], which becomes another intriguing and puzzling fundamental phenomenon in solid state physics.

At the experimental low temperature $5.45 \mathrm{~K}$ [3], excitation of optical phonons is unlikely, and thermal conduction should be carried by acoustic phonons. While $\mathrm{Tb}_{3} \mathrm{Ga}_{5} \mathrm{O}_{12}$ is an ionic material, in a perfect lattice, each unit cell is charge neutral. In the acoustic phonon modes, each unit cell vibrates as a rigid object without relative displacements between its constituting atoms [4], and does not acquire a net Lorentz force in a magnetic field. Theoretical understanding of the physical mechanism underlying the PHE is highly desirable.

In this Letter, we propose a theoretical model based upon the Raman spin-lattice interaction for the PHE. The PHE is discussed to be a phonon analogue to the AHE. The thermal Hall conductivity of the phonons in the clean limit is calculated by using the Kubo formula. The theory can explain the essential features of the experimental data for
$\mathrm{Tb}_{3} \mathrm{Ga}_{5} \mathrm{O}_{12}$, including the sign, magnitude, and linear magnetic field dependence of the thermal Hall conductivity.

We consider a sample of a paramagnetic dielectric with volume $\mathcal{V}$, which has a cubic lattice structure with symmetry axes parallel to the axes of the coordinate system. For simplicity, we assume that only one rare-earth ion in each unit cell is paramagnetic. The relevant model Hamiltonian is written as

$$
H=\sum_{q, \sigma} \hbar \omega_{q \sigma} a_{q \sigma}^{\dagger} a_{q \sigma}+V
$$

where $a_{q \sigma}^{\dagger}$ creates an acoustic phonon of wave vector $\mathbf{q}$ and polarization $\sigma$. The well-known Debye model is used to describe the acoustic phonons. We designate $\sigma=0$ for the longitudinal acoustic (LA) phonons with dispersion relation $\omega_{q 0}=c_{\mathrm{L}} q$, and $\sigma=1$ and 2 for the transverse acoustic (TA) phonons with $\omega_{q 1}=\omega_{q 2}=c_{\mathrm{T}} q . V$ represents the interaction between the phonons and the electronic spins and orbital angular momenta of the paramagnetic ions [5-10], often called the spin-lattice interaction. For $\mathrm{Tb}_{3} \mathrm{Ga}_{5} \mathrm{O}_{12}$, the rare-earth ions $\mathrm{Tb}^{3+}$ are paramagnetic with large magnetic moments and may be responsible for the spin-lattice interaction. Since microscopic calculation of the spin-lattice interaction is difficult, phenomenological description based upon symmetry considerations is usually employed [6-10]. Two most important spin-lattice interaction processes have been extensively studied in the past [5-10], i.e., the modulation interaction and the Raman interaction. The modulation interaction stands for the direct modulation of the interatomic spin-spin interaction due to lattice vibrations. The Raman interaction represents the second-order transition between the Kramers degenerate ground states of an ion through the intermediary of the excited states caused by the time-dependent variation of the crystalline field. We note that, for $\mathrm{Tb}_{3} \mathrm{Ga}_{5} \mathrm{O}_{12}$, magnetic ordered states do not occur down to very low temperature $0.2 \mathrm{~K}$ [11], suggesting that the interatomic spinspin interaction is possibly very weak. We will neglect the 
spin-spin interaction, and focus on the Raman interaction. The Raman interaction is known to dominate the spinlattice relaxation in many ionic insulators [5-8].

The electron spin-orbit coupling of a rare-earth ion is usually stronger than the crystalline field. By considering the spin-orbit coupling and intra-atomic Coulomb interaction first, the spins and orbital angular momenta of the outer-shell electrons shall form a total angular momentum $\mathbf{J}$. The ground states are a $\mathbf{J}$ multiplet, which further split in the presence of the crystalline field [6,7]. In order to develop a transparent theory, we study a relatively simple case that was often considered in the literature [6-10]. We assume that all the ground-state degeneracies of the ion except for the Kramers one are lifted by the crystalline field in such a manner that the energy difference $E_{1}$ between the lowest excited states and the ground states is greater than the Debye energy $\hbar \omega_{\mathrm{D}}$. In this case, one can obtain for the Raman interaction $[9,10] V=K_{0} \sum_{m} \mathbf{s}_{m} \cdot \boldsymbol{\Omega}_{m}$, where $K_{0}$ is a positive coupling constant, the $1 / 2$ isospin $\mathbf{s}_{m}$ describes the ground-state Kramers doublet, and $\boldsymbol{\Omega}_{m}=\mathbf{u}_{m} \times \mathbf{p}_{m}$ is the center-of-mass angular momentum of the unit cell with $\mathbf{u}_{m}$ and $\mathbf{p}_{m}$ the center-of-mass displacement and momentum. The Raman interaction is taken to be isotropic by virtue of the cubic lattice symmetry [10]. At low temperatures, where thermal excitation of the ion into the excited states is virtually impossible, the primary contribution to the magnetization comes from the ground-state doublet. The Kramers doublet carry opposite magnetic moments, which split in the presence of a magnetic field $\mathbf{B}$ and give rise to a magnetization $\mathbf{M}$. It is easy to prove that the ensemble average of the isospin is proportional to the magnetization, namely, $\left\langle\mathbf{s}_{m}\right\rangle=c \mathbf{M}$ with $c$ the proportionality coefficient [12]. Under the mean-field approximation, the Raman interaction reduces to

$$
V=K \sum_{m} \mathbf{M} \cdot \boldsymbol{\Omega}_{m},
$$

where $K=c K_{0}$, and $K M$ has the units of frequency. While Eq. (2) is obtained in the relatively simple case, it represents a minimal form of possible interaction between magnetization and phonons, and may also serve as a reasonable hypothesis for a phenomenological theory of the PHE in general paramagnetic dielectrics, similar to the theory of the AHE [1].

In order to illustrate the basic mechanism of the PHE, we consider the vibration of the $m$ th unit cell in the phonon modes of a given wave vector $\mathbf{q}$, as shown in Fig. 1. The sound speed $c_{\mathrm{T}}$ of the TA phonons is generally smaller than that of the LA phonons $c_{\mathrm{L}}$ [13], meaning that the vibration of the unit cell in the transverse directions is lower in energy than that in the longitudinal direction. This yields a correlation between the angular momentum $\boldsymbol{\Omega}_{m}$ and phonon momentum $\hbar \mathbf{q}$ that parallel and antiparallel alignments between $\boldsymbol{\Omega}_{m}$ and $\hbar \mathbf{q}$ are energetically favorable. We may regard $\boldsymbol{\Omega}_{m}$ as an internal "spin" degree of freedom of the phonons. Then the correlation between $\boldsymbol{\Omega}_{m}$ and $\hbar \mathbf{q}$ plays the role of a "spin-orbit" coupling, effectively simi-

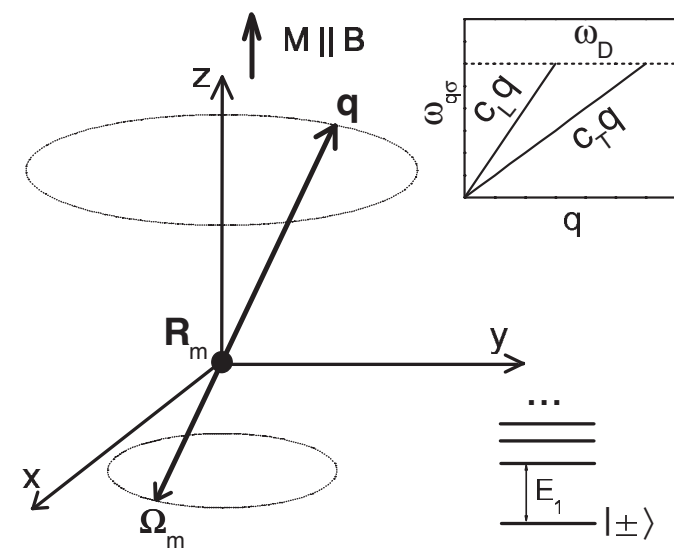

FIG. 1. Lowest-energy configuration between the phonon wave vector $\mathbf{q}$ and the center-of-mass angular momentum $\boldsymbol{\Omega}_{m}$ of a unit cell. Here, $\mathbf{R}_{m}$ represents the equilibrium position of the unit cell, which is taken to be at the origin. Top right panel shows the dispersion relations of the LA and TA phonons in the Debye model. Inset at the bottom right is a hypothetical level graph of a paramagnetic ion with $| \pm\rangle$ as the ground-state doublet.

lar to the Luttinger spin-orbit coupling [14]. Besides, the Raman interaction, Eq. (2) is a counterpart to the exchange coupling between carrier spins and magnetization in the AHE systems [1]. With these two essential ingredients, we can expect the PHE to occur in the present system, as a bosonic analogue to the AHE [1]. In Fig. 1, the lowestenergy configuration between $\boldsymbol{\Omega}_{m}$ and $\hbar \mathbf{q}$ is shown. It has been considered that, when $\mathbf{M} \neq 0$ and the Raman interaction arises as a weak perturbation, the degenerate TA modes split into two circularly polarized modes with nonvanishing $\boldsymbol{\Omega}_{m}$ parallel and antiparallel to $\hbar \mathbf{q}$, respectively, and the one with $\Omega_{m}^{z}<0$ is lower in energy. The splitting occurs in this way because $\left|\boldsymbol{\Omega}_{m}\right|$ is maximized in the circularly polarized modes, which is favorable for minimizing the Raman interaction.

We start the rigorous calculation by rewriting the Raman interaction Eq. (2) in the second-quantization representation

$$
V=\frac{1}{2} \sum_{\mathbf{q}, \sigma, \sigma^{\prime}} \Delta_{q \sigma \sigma^{\prime}} \sqrt{\frac{\omega_{q \sigma^{\prime}}}{\omega_{q \sigma}}}\left(a_{-q \sigma}+a_{q \sigma}^{\dagger}\right)\left(a_{q \sigma^{\prime}}-a_{-q \sigma^{\prime}}^{\dagger}\right),
$$

where $\Delta_{q \sigma \sigma^{\prime}}=-i \hbar K \mathbf{M} \cdot\left(\hat{\mathbf{e}}_{q \sigma}^{*} \times \hat{\mathbf{e}}_{q \sigma^{\prime}}\right)$ with $\hat{\mathbf{e}}_{q \sigma}$ the phonon polarization vector, and the phase convention $\mathbf{e}_{-q \sigma}^{*}=$ $\mathbf{e}_{q \sigma}$ is adopted. The thermal Hall conductivity $\kappa_{x y}$ can be calculated by using the Kubo formula [15]

$$
\kappa_{x y}=\frac{\mathcal{V}}{T} \int_{0}^{\hbar / k_{\mathrm{B}} T} d \lambda \int_{0}^{\infty} d t\left\langle J_{\mathrm{E}}^{x}(-i \lambda) J_{\mathrm{E}}^{y}(t)\right\rangle,
$$

where $J_{\mathrm{E}}^{x}$ is the $x$ component of the energy flux operator $\mathbf{J}_{\mathrm{E}}$ of the phonons, and $\mathbf{J}_{\mathrm{E}}(t)=e^{i H t / \hbar} \mathbf{J}_{\mathrm{E}} e^{-i H t / \hbar}$. The complete expression for $\mathbf{J}_{\mathrm{E}}$ for free phonons was derived by Hardy [16], which consists of some harmonic terms and cubic terms. In the same essence as the harmonic approximation to the real lattice Hamiltonian in the fundamental phonon 
theory [4], it is sufficient to retain the harmonic terms at low temperatures. By following the same procedure as the Eqs. (2.1) - (2.14) in Ref. [16], we derive the energy flux including the contribution from the Raman interaction Eq. (2) to be

$$
\mathbf{J}_{\mathrm{E}}=\frac{1}{2 \mathcal{V}} \sum_{m n \alpha \beta}\left(\mathbf{R}_{m}-\mathbf{R}_{n}\right) \Phi^{\alpha \beta}\left(\mathbf{R}_{m}-\mathbf{R}_{n}\right) u_{m}^{\alpha} \boldsymbol{v}_{n}^{\beta},
$$

where $u_{m}^{\alpha}$ and $\boldsymbol{v}_{m}^{\alpha}$ with $\alpha=x, y$, and $z$ are the $\alpha$ components of the center-of-mass displacement $\mathbf{u}_{m}$ and velocity $\mathbf{v}_{m}$ of the $m$ th unit cell, respectively, and $\Phi^{\alpha \beta}\left(\mathbf{R}_{m}-\mathbf{R}_{n}\right)$ are the stiffness matrix elements of the lattice with $\mathbf{R}_{m}$ the equilibrium position of the unit cell. The center-of-mass velocity is $\mathbf{v}_{m}=\mathbf{p}_{m} / M_{c}+K\left(\mathbf{M} \times \mathbf{u}_{m}\right)$ with $M_{c}$ the mass of a unit cell, where the second term originates from the Raman interaction. By using the basic relation [4] $\Phi^{\alpha \beta}(\mathbf{q})=M_{c} \sum_{\sigma}\left(\omega_{q \sigma}^{2} \hat{e}_{q \sigma}^{\alpha} \hat{e}_{q \sigma}^{* \beta}\right)$, the energy flux is derived to be $\mathbf{J}_{\mathrm{E}}=\mathbf{J}_{\mathrm{E}}^{(0)}+\mathbf{J}_{\mathrm{E}}^{(1)}$, where

$$
\mathbf{J}_{\mathrm{E}}^{(0)}=\frac{1}{2 \mathcal{V}} \sum_{\mathbf{q}, \sigma, \sigma^{\prime}} \mathbf{j}_{q \sigma \sigma^{\prime}} \sqrt{\frac{\omega_{q \sigma^{\prime}}}{\omega_{q \sigma}}}\left(a_{-q \sigma}+a_{q \sigma}^{\dagger}\right)\left(a_{q \sigma^{\prime}}-a_{-q \sigma^{\prime}}^{\dagger}\right),
$$

$$
\begin{aligned}
\mathbf{J}_{\mathrm{E}}^{(1)}= & \frac{1}{2 \mathcal{V}} \sum_{\mathbf{q}, \sigma, \sigma^{\prime}, \sigma^{\prime \prime}} \mathbf{j}_{q \sigma \sigma^{\prime \prime}}\left(\frac{\Delta_{q \sigma^{\prime \prime} \sigma^{\prime}}}{\hbar \sqrt{\omega_{q \sigma^{\prime}} \omega_{q \sigma^{\prime}}}}\right) \\
& \times\left(a_{-q \sigma}+a_{q \sigma}^{\dagger}\right)\left(a_{q \sigma^{\prime}}+a_{-q \sigma^{\prime}}^{\dagger}\right),
\end{aligned}
$$

with

$$
\begin{aligned}
\mathbf{j}_{q \sigma \sigma^{\prime}}= & \hbar \omega_{q \sigma} \delta_{\sigma \sigma^{\prime}} \nabla_{q} \omega_{q \sigma}+\frac{\hbar}{4}\left(\omega_{q \sigma}^{2}\right. \\
& \left.-\omega_{q \sigma^{\prime}}^{2}\right) \sum_{\alpha}\left[\left(\nabla_{q} \hat{e}_{q \sigma}^{* \alpha}\right) \hat{e}_{q \sigma^{\prime}}^{\alpha}-\hat{e}_{q \sigma}^{* \alpha}\left(\nabla_{q} \hat{e}_{q \sigma^{\prime}}^{\alpha}\right)\right] .
\end{aligned}
$$

Here, $\mathbf{J}_{\mathrm{E}}^{(1)}$ comes from the Raman interaction.

The energy scale $\hbar K M$ of the Raman interaction is generally much smaller than the Debye energy $\hbar \omega_{\mathrm{D}}$ as well as the average energy difference between the LA and TA phonon branches, so that a perturbation treatment of Eq. (3) suffices. Since the two TA branches are degenerate, according to the degenerate perturbation theory, we need to find suitable phonon polarization vectors, for which the direct coupling between the two TA branches $\Delta_{q 12}=$ $\Delta_{q 21}^{*}$ vanishes. Such polarization vectors are obtained as $\hat{\mathbf{e}}_{q 0}=i \hat{\mathbf{q}}, \hat{\mathbf{e}}_{q 1}=\left(\hat{\boldsymbol{\theta}}_{q}+i \hat{\boldsymbol{\varphi}}_{q}\right) / \sqrt{2}$, and $\hat{\mathbf{e}}_{q 2}=\left(\hat{\boldsymbol{\theta}}_{q}-i \hat{\boldsymbol{\varphi}}_{q}\right) / \sqrt{2}$, where $\hat{\mathbf{q}}, \hat{\boldsymbol{\theta}}_{q}$, and $\hat{\boldsymbol{\varphi}}_{q}$ are the unit vectors associated with wave vector $\mathbf{q}=\left(q, \theta_{q}, \varphi_{q}\right)$ in the spherical polar coordinate system. We notice that $\hat{\mathbf{e}}_{q 1}\left(\hat{\mathbf{e}}_{q 2}\right)$ for the TA phonons is a superposition of two orthogonal linear polarization vectors $\hat{\boldsymbol{\theta}}_{q}$ and $\hat{\boldsymbol{\varphi}}_{q}$ with a fixed phase shift $90^{\circ}\left(-90^{\circ}\right)$ between them. This indicates that the TA phonons indeed split into two circularly polarized branches upon the perturbation of the spin-lattice interaction. The standard nondegenerate perturbation theory is then applied to calculate the many- particle eigenstates of the system to the linear order in the Raman interaction. By substitution of the eigenstates into Eq. (4), we consequently obtain for the thermal Hall conductivity

$$
\kappa_{x y}=\frac{\gamma k_{\mathrm{B}} K M}{2 \pi^{2} \bar{c}_{s}}\left(\frac{k_{\mathrm{B}} T}{\hbar}\right) \int_{0}^{\Theta_{\mathrm{D}} / T} \frac{x}{e^{x}-1} d x,
$$

where $\gamma=(5-\delta)(1+\delta)^{4} /\left[4 \delta^{2}\left(9+18 \delta^{3}\right)^{1 / 3}\right]$ with $\delta=$ $c_{\mathrm{L}} / c_{\mathrm{T}}, \bar{c}_{s}$ is the average sound speed defined by $3 / \bar{c}_{s}^{3}=$ $\left(1 / c_{\mathrm{L}}^{3}+2 / c_{\mathrm{T}}^{3}\right)$ and $\Theta_{\mathrm{D}}=\hbar \omega_{\mathrm{D}} / k_{\mathrm{B}}=\left(6 \pi^{2} / \nu_{0}\right)^{1 / 3} \hbar \bar{c}_{s} / k_{\mathrm{B}}$ is the Debye temperature with $\nu_{0}$ the volume of a unit cell.

In Fig. 2, the calculated $\kappa_{x y}$ at $T=5.45 \mathrm{~K}$ is plotted as a function of $K M / \omega_{\mathrm{D}}$ for two different values of $\delta$. Here, we set $\hbar \omega_{\mathrm{D}}=0.05 \mathrm{eV}$ and $\nu_{0}=(12 \AA)^{3}$, which correspond to $\Theta_{\mathrm{D}} \simeq 580 \mathrm{~K}$ and $\bar{c}_{s} \simeq 850 \mathrm{~m} / \mathrm{s}$. These are either known values for $\mathrm{Tb}_{3} \mathrm{Ga}_{5} \mathrm{O}_{12}$ or typical values for rare-earth garnets with similar structures [11]. It is clear that the thermal Hall conductivity $\kappa_{x y}$ is linear in the magnetization $M$. When the applied magnetic field $B$ is relatively weak, $M$ varies linearly with $B$, and so does $\kappa_{x y}$. We can expect that, while $\kappa_{x y}$ remains linear as a function of $M$, it may possibly become nonlinear as a function of $B$ at very strong magnetic field. It is worthwhile to test this prediction in experiment. In the inset of Fig. 2, $\kappa_{x y}$ as a function of $\delta$ is plotted. $\kappa_{x y}$ decreases with increasing $\delta$. From Eq. (9), we see that $\kappa_{x y}$ will change sign at $\delta=5$. We notice that Eqs. (6) and (7) make comparable opposite contributions to $\kappa_{x y}$. Their competition accounts for the nontrivial dependence of $\kappa_{x y}$ on $\delta$. In most materials, the typical values of $\delta$ are around 2 [13], where $\kappa_{x y}$ is always positive.

In Fig. 3, $k_{x y}$ for a fixed value of $K M$ is shown as a function of normalized temperature $T / \Theta_{\mathrm{D}}$. At low temperatures $T \ll \Theta_{\mathrm{D}}$, we can obtain from Eq. (9) $\kappa_{x y} \simeq$ $\left(1.64 \gamma k_{\mathrm{B}} K M / 2 \pi^{2} \bar{c}_{s}\right)\left(k_{\mathrm{B}} T / \hbar\right)$, where the numerical identity $\sum_{n \geq 1} n^{-2} \simeq 1.64$ has been used. From Fig. 3, we see that the above linear dependence of $\kappa_{x y}$ on temperature is

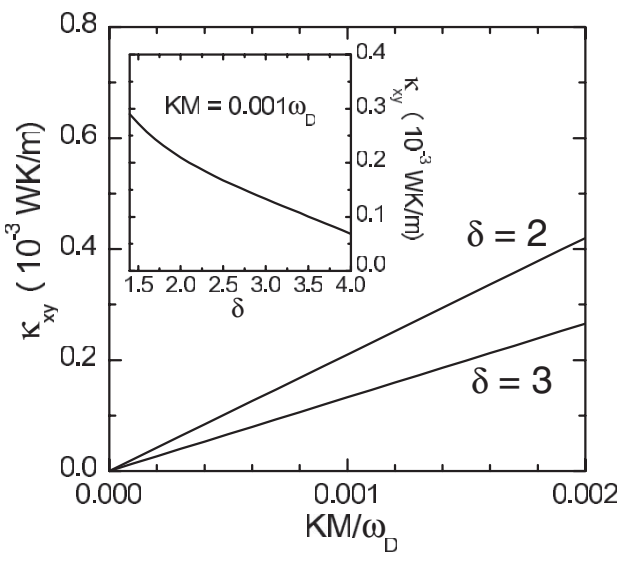

FIG. 2. Thermal Hall conductivity $\boldsymbol{\kappa}_{x y}$ as a function of $K M / \omega_{\mathrm{D}}$ for two different values of $\delta=c_{\mathrm{L}} / c_{\mathrm{T}}$. Inset: $\kappa_{x y}$ as a function of $\delta$ for $K M=0.001 \omega_{\mathrm{D}}$. Here, $T=5.45 \mathrm{~K}, \hbar \omega_{\mathrm{D}}=$ $0.05 \mathrm{eV}$ and $\nu_{0}=(12 \AA)^{3}$. 


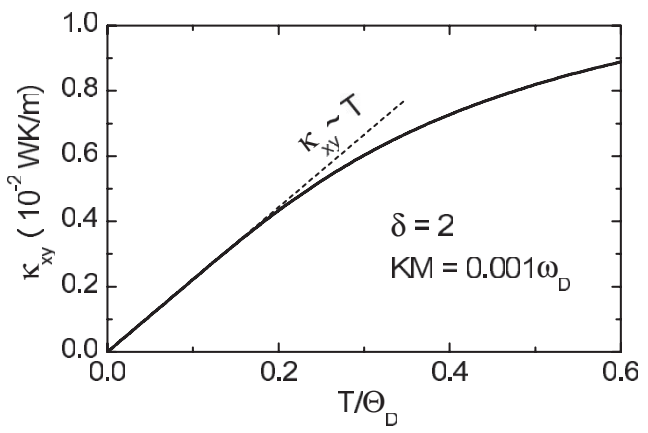

FIG. 3. $\kappa_{x y}$ as a function of normalized temperature for $K M=$ $0.001 \omega_{\mathrm{D}}$ and $\delta=2$ (solid line). Other parameters are chosen to be the same as in Fig. 2. The dashed line is a linear fit to $\kappa_{x y}$ at low temperatures.

well valid for $T \leqq 0.2 \Theta_{\mathrm{D}}$. From an experimental point of view, it is relatively convenient to consider the fixed magnetic field instead of fixed magnetization. For paramagnetic materials and a relatively weak magnetic field, we may use the Curie's law $M \propto B / T$. As a result, at fixed magnetic field, $\kappa_{x y}$ approaches a constant at low temperatures. At relatively high temperatures $T \geqslant 0.2 \Theta_{\mathrm{D}}$, it decreases with increasing temperature.

We can show that the present theory reproduces the essential characteristics of the experimental data [3]. In the experiment [3], a constant longitudinal heat current is driven through the sample, and the temperature difference $\Delta T$ in the transverse direction across the sample is measured. First, the present theory predicts $\kappa_{x y}>0$ for typical values of $\delta$, meaning that, if the magnetic field is along the $z$ axis and a heat current is driven along the $x$ axis, the Hall heat current will flow along the $y$ axis, which is consistent with the experimental relation among the sign of $\Delta T$ and the directions of the magnetic field and the driving heat current [3]. Second, $\Delta T$ is observed to be linear in the magnetic field $B$ [3]. By analogy with the charge Hall effect, $\Delta T$ is proportional to the thermal Hall resistivity $r_{x y}=\kappa_{x y} /\left(\kappa_{x x}^{2}+\kappa_{x y}^{2}\right) \simeq \kappa_{x y} / \kappa_{x x}^{2}$, where the longitudinal thermal conductivity $\kappa_{x x}$ depends weakly on $B$ [3]. It follows that the experimental thermal Hall conductivity $\kappa_{x y} \propto B$. In the present theory, $\kappa_{x y} \propto B$ at relatively weak magnetic field, which agrees with the experimental observation. Third, if $K M$ is taken to be about $10^{-4} \omega_{\mathrm{D}}$, we see from Fig. 2 that $\kappa_{x y}$ is $10^{-5}-10^{-4} \mathrm{~W} \mathrm{~K} / \mathrm{m}$, which will be comparable to the experimental value $4.5 \times 10^{-5} \mathrm{~W} \mathrm{~K} / \mathrm{m}$ at $B=1 \mathrm{~T}$ and $T=5.45 \mathrm{~K}$. Here, the experimental value of $\kappa_{x y}$ is deduced from the reported thermal conductivity $\kappa_{x x}=4.5 \times 10^{-1} \mathrm{~W} \mathrm{~K} / \mathrm{m}$ and Hall angle $\kappa_{x y} / \kappa_{x x}=1 \times$ $10^{-4} \mathrm{radT}^{-1}$ [3]. To estimate the Raman coupling strength, we can use the relation $K M=K_{0}\left|\left\langle\mathbf{s}_{m}\right\rangle\right|$. For the experimental material, while the average of the isospin is affected by many factors, such as the actual crystalline field and multiple paramagnetic ions in a unit cell, we may conservatively assume that the effective $\left|\left\langle\mathbf{s}_{m}\right\rangle\right|$ is of the order of $0.1 \hbar$ at $B=1 \mathrm{~T}$ and $T=5.45 \mathrm{~K}$, where the Zeeman energy is already comparable to $k_{\mathrm{B}} T$. Thus $K_{0}$ is estimated about $K_{0} \simeq 10^{-3} \omega_{\mathrm{D}} / \hbar$ or $\hbar^{2} K_{0} \simeq 1 \mathrm{~cm}^{-1}$, which can be found to be within the possible range of the Raman coupling strength in ionic insulators [6,7].

This work is supported by ACS-PRF 41752-AC10, Research Corporation Fund CC5643, the NSF Grant No. DMR-0307170 (DNS), and a grant from the Robert A. Welch Foundation under the Grant No. E-1146 (CST).

[1] T. Jungwirth, Q. Niu, and A. H. MacDonald, Phys. Rev. Lett. 88, 207208 (2002); Z. Fang et al., Science 302, 92 (2003); F. D. M. Haldane, Phys. Rev. Lett. 93, 206602 (2004).

[2] S. Murakami, N. Nagaosa, and S. C. Zhang, Science 301, 1348 (2003); J. Sinova et al., Phys. Rev. Lett. 92, 126603 (2004).

[3] C. Strohm, G. L. J. A. Rikken, and P. Wyder, Phys. Rev. Lett. 95, 155901 (2005).

[4] M. P. Marder, Condensed Matter Physics (John Wiley \& Sons, New York, 2000).

[5] R. de L. Kronig, Physica (Amsterdam) 6, 33 (1939); J. H. Van Vleck, Phys. Rev. 57, 426 (1940); R. D. Mattuck and M. W. P. Strandberg, Phys. Rev. 119, 1204 (1960).

[6] R. Orbach, Proc. R. Soc. A 264, 458 (1961).

[7] Spin-Lattice Relaxation in Ionic Solids, edited by A. A. Manenkov and R. Orbach (Harper \& Row, New York, 1966).

[8] A. Abragam and B. Bleaney, Electron Paramagnetic Resonance of Transition Ions (Oxford University, London, 1970).

[9] T. Ray and D. K. Ray, Phys. Rev. 164, 420 (1967).

[10] H. Capellmann and S. Lipinski, Z. Phys. B 83, 199 (1991); A. S. Ioselevich and H. Capellmann, Phys. Rev. B 51, 11446 (1995).

[11] G. A. Slack and D. W. Liver, Phys. Rev. B 4, 592 (1971).

[12] By assumption, $E_{1}$ should be much greater than the Zeeman energy, and so the Zeeman energy admixtures of excited states into the ground-state doublet are negligible. The $z$ axis is chosen as the quantization axis for $\mathbf{J}$ and $\mathbf{s}_{m} . J^{z}$ projected into the subspace spanned by the doublet can be written in the general form $J^{z}=$ $\xi_{0}+\xi_{1}\left(\mathbf{s}_{m} \cdot \hat{\mathbf{e}}\right)$, where $\xi_{0}$ and $\xi_{1}$ are two constants. Because of time-reversal symmetry, $\xi_{0}$ actually vanishes. By considering cubic lattice symmetry, the unit vector $\hat{\mathbf{e}}$ is found to be along the $z$ axis, and hence $J^{z}=\xi_{1} s_{m}^{z}$. Thus $M^{z}=\left\langle g_{\mathrm{J}} \mu_{\mathrm{B}} J^{z}\right\rangle / \nu_{0}=g_{\mathrm{J}} \mu_{\mathrm{B}} \xi_{1}\left\langle s_{m}^{z}\right\rangle / \nu_{0}$ with $\nu_{0}$ the volume of a unit cell. Since $\mathbf{M}$ and $\left\langle\mathbf{s}_{m}\right\rangle$ are both parallel to the $z$ axis by symmetry, we can also write $\left\langle\mathbf{s}_{m}\right\rangle=c \mathbf{M}$ with $c=\nu_{0} / g_{\mathrm{J}} \mu_{\mathrm{B}} \xi_{1}$.

[13] M. de Podesta, Understanding the Properties of Matter (Taylor \& Francis, New York, 2002).

[14] J. M. Luttinger, Phys. Rev. 102, 1030 (1956).

[15] G. D. Mahan, Many-Particle Physics (Plenum, New York, 1990).

[16] R. J. Hardy, Phys. Rev. 132, 168 (1963). 